CERN-TH.6395/92

SISSA $31 / 92 / \mathrm{EP}$

\title{
PLANCKIAN SCATTERING BEYOND THE SEMICLASSICAL APPROXIMATION
}

\author{
D. Amati \\ International School for Advanced Studies, Trieste \\ and \\ INFN, Sezione di Trieste, Italy \\ M. Ciafaloni \\ Dipartimento di Fisica, Universitá di Firenze \\ and \\ INFN, Sezione di Firenze, Italy \\ G. Veneziano \\ CERN, Geneva, Switzerland
}

\begin{abstract}
Motivated by recent investigations of near-forward Planckian energy collisions, we compare various approaches giving the same leading eikonal approximation. We stress the need of going beyond this approximation in order to control impact parameters $b \simeq$ $G E$ which are crucial for understanding $S$-matrix singularities ('t Hooft poles) and the physics of gravitational collapse (black hole formation). We point out a few problems that alternative methods have to tackle in order to improve on the direct calculations that we have discussed elsewhere.
\end{abstract}

CERN-TH.6395/92

SISSA $31 / 92 / \mathrm{EP}$

February 1992 



\section{Introduction}

A revival of interest has recently arisen in the study of small angle gravitational scattering at Planckian energies $\sqrt{s} \equiv 2 E \geq M_{p}$. This regime is characterized by a strong effective coupling $\alpha_{G} \equiv \frac{G s}{\hbar} \geq 1$ which makes any simple perturbative expansion unwarranted.

Different approaches have been proposed in the last few years ${ }^{(1-5)}$ which take into account all orders in $\alpha_{G}$. In this note we will show how and why they all coincide in the leading approximation, which has a semiclassical effective metric interpretation, while most of them fail in providing the non-leading terms under which new classical and quantum gravity effects are hiding.

To be more precise, leading contributions to the scattering at a given impact parameter $b=J / E$ contain all powers of $\alpha_{G}$. The non-leading ones contain additional powers of the Newton constant $G$ in the two dimensionless combinations*)

$$
\frac{\hbar G}{b^{2}}=\frac{\lambda_{p}^{2}}{b^{2}}, \quad \frac{G^{2} s}{b^{2}}=\frac{R^{2}}{b^{2}}=\alpha_{G} \frac{\lambda_{p}^{2}}{b^{2}},
$$

which are small as long as $b$ is much larger than the Planck length $\lambda_{p}$ and the gravitational radius $R=2 G E$.

The leading result for the $S$ matrix $S(b, E)$ - valid for $b \gg \lambda_{p}, R$ - is the same in all approaches and has the semiclassical form

$$
\begin{aligned}
& S(b, E)=e^{2 i \delta_{0}(b, E)}, \\
& \delta_{0}(b, E)=-\frac{G s}{\hbar} \log b,
\end{aligned}
$$

where we have omitted, as usual, the unobservable, infrared-singular Coulomb phase.

Let us comment briefly on each one of these approaches**)

't Hooft ${ }^{(1)}$ obtained this result by solving for the scattering amplitude of one particle, seen as a test body, in the background metric of the other particle, an Aichelburg-Sexl ${ }^{(6)}$ metric.

Muzinich and Soldate ${ }^{(2)}$ obtained an equivalent result by an eikonal approximation to reggeized graviton exchange and recognized that, for $b$ approaching $R$, the $b$-representation fails in general to diagonalize and fulfill unitarity.

*) In the case of string theory, quantum string corrections of relative order $\frac{\hbar \alpha^{\prime}}{b^{2}} \equiv \frac{\lambda_{s}^{2}}{b^{2}}$ are also present, and have been computed ${ }^{(3,4)}$.

**) We follow the order of submission, noting that the first three papers were submitted within ten days in July 1987 ! 
Our derivation ${ }^{(3)}$ was based on the resummation of the string loop expansion at high energies. This gave the leading result, Eq.(1), and allowed the identification and computation of corrections in $\lambda_{s} / b^{(3)}$ and in $R / b^{(4)}$. The semiclassical relation between impact parameter and scattering angle also suggested, at leading level, the interpretation in terms of an effective Aichelburg-Sexl metric.

In a recent paper ${ }^{(5)}$, E. and $H$. Verlinde reobtained the leading result of Eq.(1) by studying the Einstein action in a gauge that separates $g_{\mu \nu}$ in longitudinal and transverse components with respect to the beam direction. In this gauge, they split the action in two terms: the first one can be treated classically at high energies, while the second, although exhibiting a strong coupling, can be dealt with thanks to its topological structure in the two longitudinal directions.

\section{The eikonal amplitude}

In order to understand better the leading approximation, one would like to rewrite it in the momentum transfer $t=-q^{2}$ representation. In principle, this requires knowledge of the amplitude $S(\underline{b}, E)$ for all impact parameters, including the dangerous region $b \leq R$.

Fortunately, the latter region is irrelevant at small angles $\theta^{2}=\frac{q^{2}}{s} \ll 1$, for which the most important contribution comes from $b \simeq \frac{R}{\theta} \gg R$. Therefore, the small angle amplitude can be safely obtained either by a saddle point method ${ }^{(3)}$ or, more simply, by extending $^{(1)}$ up to $b=0$ the eikonal expression (1). In this last way one obtains

$$
\begin{gathered}
\frac{1}{s} A^{e i k}\left(s,-\mathbf{q}^{2}\right)=4 \int d^{2} b e^{i \mathbf{b} \cdot \mathbf{q}} \frac{e^{2 i \delta_{0}(b, E)}}{2 i}= \\
=\frac{8 \pi \alpha_{G}}{\mathbf{q}^{2}}\left(\frac{4}{\mathbf{q}^{2}}\right)^{-i \alpha_{G}} \frac{\Gamma\left(1-i \alpha_{G}\right)}{\Gamma\left(1+i \alpha_{G}\right)} \quad\left(q=2 E \sin \frac{\theta}{2}\right) .
\end{gathered}
$$

The saddle point evaluation yields the same result, apart from the $\Gamma$ functions ratio, which for real $s$ is a $q$-independent, hence irrelevant, phase factor.

It is interesting to notice that, for pure graviton exhange in four dimensions, the $b$ transform - with the above definition of $q$ - diagonalizes unitarity, as proven in Appendix $\mathrm{B}$ of ref.4. The alternative definition of $q$ used in ref.2 explains the different unitarity properties of low partial waves in these two approaches to the leading eikonal. Thus, if the dynamics only comes from generalized ladders of pure gravitons, Eq. (2) is valid at all angles, a property of Coulomb-like interactions known to experts ${ }^{(7)}$.

This observation is confirmed by the partial wave projection of Eq.(2), which yields:

$$
\frac{i}{8 \pi} A_{J}^{e i k}(s)=e^{2 i \delta_{J}^{e i k}}=\left(\frac{s}{4}\right)^{i \alpha_{G}} \frac{\Gamma\left(J+1-i \alpha_{G}\right)}{\Gamma\left(J+1+i \alpha_{G}\right)},
$$


expression that coincides, up to a $J$-independent phase, with the ultra-relativistic limit of the relativistic Coulomb amplitude (without fine structure corrections)

$$
e^{2 i \delta_{J}^{\text {Coul }}}=\frac{\Gamma\left(J+1-i \frac{E}{k} \alpha\right)}{\Gamma\left(J+1+i \frac{E}{k} \alpha\right)}, \quad k=\sqrt{E^{2}-m^{2}},
$$

after replacing $\alpha$ by $\alpha_{G}$.

The above coincidence allows an interpretation of the 't Hooft poles of $A^{e i k}$ at $i \alpha_{G}=$ $i \frac{G s}{\hbar}=N=1,2, \ldots$. They correspond to the $J$-plane poles of Eq.(3) at $J=i \alpha_{G}-n-1$ and are thus nothing but the $k \rightarrow \infty$ limit of the Regge trajectories $J_{n}(k)=-n-1+i \alpha \frac{E}{k}$, interpolating the Coulomb bound states at $k_{n}=\frac{i \alpha m}{J+n+1}\left(1+0\left(\frac{\alpha^{2}}{J^{2}}\right)\right)$.

Note that the 't Hooft poles originate from the $b \rightarrow 0$ singularity of $A^{e i k}(b, E)$ in Eq.(1), and thus from the short distance behaviour of the Coulomb interaction. If this is softened, as for instance in the string approach, the poles disappear ${ }^{(3)}$. A simple illustration of this fact is given by the replacement

$$
\delta_{0} \rightarrow \widetilde{\delta}_{0}=-\frac{1}{2} \alpha_{G} \log \left(b^{2}+b_{0}^{2}\right),
$$

which yields

$$
\frac{1}{s} \widetilde{A}^{e i k}\left(s,-\mathbf{q}^{2}\right)=\frac{16 \pi \alpha_{G}}{\Gamma\left(1+i \alpha_{G}\right)}\left(\frac{q}{2 b_{0}}\right)^{i \alpha_{G}-1} K_{1-i \alpha_{G}}\left(b_{0} q\right),
$$

which is an entire function of $\alpha_{G}$. Eqs.(6) and (2) have the same behaviour in the small angle region $\alpha_{G} \gg b_{0} q$, i.e. if the saddle point value of $b$ exceeds $b_{0}$. By contrast, for $i \alpha_{G}=1, \widetilde{A}^{e i k}$ is finite at finite $q b_{0}$.

In conclusion, we stress the crucial importance of understanding the $b=0(R)$ region in order to reach finite angles and to study the singularity structure of the amplitude at large energies.

\section{Beyond the eikonal}

In our previous work ${ }^{(3,4)}$ we have computed the first nonleading contributions, which could be embodied in a generalized eikonal representation for the elastic $S$ matrix

$$
S(b, E)=e^{2 i\left(\delta_{0}+\delta_{1}+\delta_{2}+\ldots\right)},
$$

where $\frac{\delta_{1}}{\delta_{0}}=0\left(\frac{\lambda_{p}^{2}}{b^{2}}\right)$ and $\frac{\delta_{2}}{\delta_{0}}=0\left(\frac{R^{2}}{b^{2}}\right)$. We found ${ }^{(4)}$, for pure gravity,

$$
\delta_{1}=\frac{6}{\pi} \frac{G^{2} s}{b^{2}} \log s, \quad \operatorname{Re} \delta_{2}=2 \frac{G^{3} s^{2}}{\hbar b^{2}} .
$$


As discussed before, $\delta_{0}$ represents the physics of pure graviton exchange (i.e. the generalized ladders of Fig.1). On the other hand $\delta_{1}$ originates from the one-loop radiative corrections of the type shown in Fig.2 and $\delta_{2}$ from the " $H$ diagram" of Fig.3 and other two-loop contributions. This last correction is important in the "classical" regime $R \gg \lambda_{p}$ : it shows an increase of the Einstein deflection, and thus of the gravitational interaction, for $b \sim R$. This may be the first sign of a gravitational instability that could build-up from the resummation of higher orders in $\frac{R^{2}}{b^{2}}$.

From the metric point of view, $\delta_{0}$ comes from semiclassical scattering in the Aichelburg-Sexl metric ${ }^{(6)}$

$$
d s^{2}=d x_{+} d x_{-}-d \mathbf{x}^{2}+4 G E \delta\left(x_{+}\right)\left(d x_{+}\right)^{2} \log \mathbf{x}^{2}
$$

where $\mathbf{x}=\left(x_{1}, x_{2}\right)$ are the transverse coordinates and $x_{ \pm}=x_{0} \pm x_{3}$ the longitudinal ones.

On the other hand, $\delta_{1}$ and $\delta_{2}$ are related to gravitational emission and thus cannot be directly interpreted in terms of an external metric. Rather, they provide a rough estimate of the quantum fluctuations around the metric (9), as follows

$$
\left\langle(\delta g)^{2}\right\rangle \simeq \frac{\delta_{1}}{\delta_{0}} \simeq \frac{\delta_{2}}{\delta_{0}^{2}} \simeq \frac{\lambda_{p}^{2}}{b^{2}}
$$

A more precise description of graviton emission associated with gravitational scattering (Fig.3) is obtained by a suitable generalization ${ }^{(4,8,9)}$ of Weinberg's soft graviton formula. Choosing the two physical (transverse, traceless) polarizations

$$
\varepsilon_{\mu \nu}^{(a)}=\frac{1}{\sqrt{2}}\left(\varepsilon_{\mu}^{L} \varepsilon_{\nu}^{T}+\varepsilon_{\mu}^{T} \varepsilon_{\nu}^{L}\right), \quad \varepsilon_{\mu \nu}^{(b)}=\frac{1}{\sqrt{2}}\left(\varepsilon_{\mu}^{L} \varepsilon_{\nu}^{L}-\varepsilon_{\mu}^{T} \varepsilon_{\nu}^{T}\right),
$$

where the vectors $\varepsilon_{\mu}^{L}\left(\varepsilon_{\mu}^{T}\right)$ are longitudinal (transverse) to the beam and orthogonal to the graviton mementum $q_{\mu}$, we find that both are emitted, with amplitudes in the ratio

$$
A^{(b)} / A^{(a)}=\tan \theta_{12}
$$

Here $\mathbf{q}_{1}, \mathbf{q}_{2}$ are the momentum transfers defined in Fig. 3, with $\mathbf{q}=\mathbf{q}_{1}+\mathbf{q}_{2}$. In the soft limit $\mathbf{q} \rightarrow 0$ the ratio (12) vanishes, showing that polarization $(a)$ contains the dominant infrared singularity, in agreement with Weinberg's results. We have seen ${ }^{(4)}$ how such a singularity has a Bloch-Nordsieck interpretation and cancels in computing $\operatorname{Re} \delta_{2}$, which gets finite contributions from both polarizations.

Admittedly, our method for computing non-leading terms is as tedious and cumbersome as a typical higher order field theory calculation. This makes it unlikely that we will 
be able to go beyond the present two-loop stage. It is thus natural to ask whether the other approaches can be extended in any simple way in order to investigate non-leading corrections. We shall illustrate why, unfortunately, this is not the case, at least for the most naive extensions.

We have already discussed the inadequacy of the external metric approach ${ }^{(1)}$ in dealing with the non-linear graviton interactions, which are responsible for corrections involving powers of $\frac{R^{2}}{b^{2}}=\left(\frac{G s}{J}\right)^{2}$.

At first sight, Verlinde's ${ }^{(5)}$ approach of separating longitudinal and transverse degrees of freedom through a gauge choice appears more promising. Unfortunately, a careful analysis shows that the salient features of the quantum fluctuations cannot be handled without modifying that framework.

In fact, the leading IR polarization $\varepsilon^{(a)}$, which is crucial for determining both $\operatorname{Im} \delta_{2}$ and $\operatorname{Re} \delta_{2}$ cannot be described in the Verlinde gauge $g_{L T}=0$. Metric fluctuations of the type $\varepsilon_{\mu \nu}^{(a)}$, which are necessary to describe a real emission process, cannot be brought to block-diagonal form by any diffeomorphism $\delta g_{\mu \nu}=\xi_{\mu} q_{\nu}+\xi_{\nu} q_{\mu}$ because $\sum_{L T} \delta g_{L T} \varepsilon_{L T}^{(a)}=0$.

This observation can be traced back to the fact that, at least around flat space time, the $g_{L T}=0$ gauge has a non-trivial zero mode $\xi_{L}=q_{L}, \xi_{T}=-q_{T}$. Thus diffeomorphisms have three essential parameters, instead of four. The fact that this gauge is not appropriate for describing fluctuations around flat space-time has already been noted in Ref.(5). Our point here is that precisely these fluctuations determine the value of non-leading corrections.

Furthermore, the second physical polarization $\varepsilon^{(b)}$, which is already block-diagonal, has equal $L L$ and TT amplitudes in contrast with the idea that the corresponding quantum fluctuations have different scales.

Finally, even if one could reduce the quantum problem to an effective action with two couplings of different magnitudes $g_{/ /}^{2}=\frac{\lambda_{p}^{2}}{\ell_{/ /}^{2}} \simeq \alpha_{G}, g_{\perp}^{2}=\frac{\lambda_{p}^{2}}{\ell_{\perp}^{2}} \simeq \frac{\lambda_{p}^{2}}{b^{2}}$, the two dynamics could not be disentangled in order to produce the subleading correction $\frac{\delta_{2}}{\delta_{0}} \simeq \frac{R^{2}}{b^{2}} \simeq g_{/ /}^{2} g_{\perp}^{2}$.

In conclusion, while we think that the crucial difference between longitudinal and transverse modes is present in the semiclassical (leading) approximation - only longitudinal gravitons coupled to the fast external sources - we see that all modes contribute on an equal footing to the higher order interactions which are responsible for the physics at $b \sim O(R)$.

We still hope that an effective eikonal approach could be constructed also for the nonleading contributions. Perhaps, the shock-wave nature of the classical field may be used to define an effective action for the longitudinal modes, in which quantum fluctuations 
of the other components are integrated out (for a step in this direction, see Ref.10). To do so, however, we first have to cope with an all-order definition of the asymptotic states (infrared gravitons) and we also have to treat all metric fluctuations on the same footing.

\section{References}

1. G.'t Hooft, Phys. Lett. 198B(1987)61.

2. I.Muzinich and M.Soldate, Phys. Rev. D37(1988)353.

3. D. Amati, M. Ciafaloni and G. Veneziano, Phys. Lett. 197B(1987)81; Int. J. Mod. Phys. A3(1988)1615.

4. D. Amati, M. Ciafaloni and G. Veneziano,Nucl. Phys. B347(1990)550.

5. E. Verlinde and H. Verlinde, Scattering at Planckian Energies, Princeton preprint PUTP-1279 (1991); see also R. Kallosh, Geometry of Scattering at Planckian Energies, Stanford preprint SU-ITP 903 (1991).

6. P. C. Aichelburg and R. U. Sexl, Gen. Rel. Grav. 2(1971)303.

7. J. D. Jackson, Phys. Lett. 215B(1988)151.

8. L. N. Lipatov, Sov. Phys. JETP. 55(1982)582; Nucl. Phys. B307(1988)705.

9. M. Ademollo, A. Bellini and M. Ciafaloni, Phys. Lett. B223(1989)318; Nucl. Phys. B338(1990)114.

10. L. N. Lipatov, Nucl. Phys. B365(1991)614.

\section{Figure Captions}

Fig.1 Graviton-exchange diagrams providing the leading eikonal approximation.

Fig.2 A typical diagram giving a quantum correction to the eikonal.

Fig.3 Diagrams giving the lowest order classical correction to the eikonal. 


$$
\frac{\frac{I X I X}{I}}{\frac{I}{1}}
$$

